\title{
All-optical WDM Regeneration of DPSK Signals using Optical Fourier Transformation and Phase Sensitive Amplification
}

Guan, Pengyu; Røge, Kasper Meldgaard; Kjøller, Niels-Kristian; Mulvad, Hans Christian Hansen; Hu, Hao; Galili, Michael; Morioka, Toshio; Oxenløwe, Leif Katsuo

\section{Published in:}

Proceedings of ECOC 2015

Link to article, DOI:

10.1109/ECOC.2015.7342010

Publication date:

2015

Document Version

Peer reviewed version

Link back to DTU Orbit

Citation (APA):

Guan, P., Røge, K. M., Kjøller, N-K., Mulvad, H. C. H., Hu, H., Galili, M., Morioka, T., \& Oxenløwe, L. K. (2015). All-optical WDM Regeneration of DPSK Signals using Optical Fourier Transformation and Phase Sensitive Amplification. In Proceedings of ECOC 2015 IEEE. https://doi.org/10.1109/ECOC.2015.7342010

\section{General rights}

Copyright and moral rights for the publications made accessible in the public portal are retained by the authors and/or other copyright owners and it is a condition of accessing publications that users recognise and abide by the legal requirements associated with these rights.

- Users may download and print one copy of any publication from the public portal for the purpose of private study or research.

- You may not further distribute the material or use it for any profit-making activity or commercial gain

- You may freely distribute the URL identifying the publication in the public portal 


\title{
All-optical WDM Regeneration of DPSK Signals using Optical Fourier Transformation and Phase Sensitive Amplification
}

\author{
P. Guan, K. M. Røge, N. K. Kjøller, H. C. H. Mulvad, H. Hu, M. Galili, T. Morioka, L. K. Oxenløwe
}

DTU Fotonik, Department of Photonics Engineering, Technical University of Denmark, Ørsteds Plads, Building 343, DK-2800 Kgs. Lyngby, Denmark, pengu@fotonik.dtu.dk

*Now with Optoelectronics Research Centre, University of Southampton, Southampton, SO17 1BJ, UK

Abstract We propose a novel all-optical WDM regeneration scheme for DPSK signals based on optical Fourier transformation and phase sensitive amplification. Phase regeneration of a WDM signal consisting of $4 \times 10-$ Gbit/s phase noise degraded DPSK channels is demonstrated for the first time.

\section{Introduction}

All-optical regeneration of wavelength division multiplexed (WDM) signals could play an important role in future optical communication systems. Although various solutions have been demonstrated using self-phase modulation effects in highly nonlinear fibers, they are limited to amplitude modulated signals ${ }^{1}$. Modern communication systems use both amplitude and phase modulation to achieve higher spectral efficiencies and higher total capacities. Recently, phase sensitive amplifiers (PSAs) have emerged as a promising all-optical way to regenerate phase-encoded signals. However only single channel optical regeneration has been demonstrated for differential phase-shift keying (DPSK) and quadrature phase-shift keying $(\text { QPSK) })^{2}$. It is important to extend PSA schemes to WDM regeneration. However, such WDM regeneration has not been demonstrated yet. The main challenge lies in keeping multiple pumps and the data signal carriers in-phase at the PSA input, which is not possible for WDM channels with random phase carriers.

In this paper, we propose a novel all-optical WDM regeneration scheme for DPSK signals based on optical Fourier transformation (OFT) and phase sensitive amplification. OFT, also known as the optical time lens principle has been shown to be a very powerful tool for manipulating the shape of optical signals in and between the time and frequency domains ${ }^{3}$. Here, OFT is used for converting the WDM signal to an optical time division multiplexed (OTDM) signal, where the individual channels are separated in time, not in wavelength, and hence easy to regenerate using a PSA. Using this scheme, we simultaneously regenerate four 10-Gbit/s phase noise degraded incoherent DPSK WDM channels. The power penalty is improved by $1.3 \mathrm{~dB}$ at $\mathrm{BER}=10^{-3}$. Forward error correction (FEC) is employed, resulting in errorfree performance for all regenerated WDM channels. To the best of our knowledge, this is the first demonstration of all-optical DPSK-WDM phase regeneration.

\section{Principle}

The main idea of the proposed scheme is to convert all DPSK WDM channels to a phase coherent OTDM $(\text { PC-OTDM })^{4}$ signal intermediately using OFT and cross-phase modulation (XPM), then regenerate the PCOTDM tributaries simultaneously in a single PSA, and finally convert it back to WDM channels. The principle of all-optical WDM regeneration of DPSK signals is shown in Fig. 1. A number of WDM DPSK channels $(\mathbf{A})$ with channel spacing $\Delta v$ are passed through a 1 bit delay interferometer (DI) and converted to OOK signals (B). To achieve multi-channel conversion in the $\mathrm{DI}, \Delta \boldsymbol{v}$ is accurately set to an integer multiple of the symbol rate. An OFT composed of quadratic phase modulation with chirp rate $K$ and a dispersion medium with dispersion $D$, is employed for WDM to OTDM conversion. After the OFT, the WDM signal is thus converted to an incoherent OTDM OOK signal with tributary spacing given by $\Delta t=2 \pi \Delta v / K$ (C). XPM in a highly nonlinear fiber (HNLF) is employed to transfer the data modulation from the OOK signal to the phase of a continuous wave $(\mathrm{CW})$ carrier (D), resulting in a PC-OTDM DPSK signal (E). After combination with two

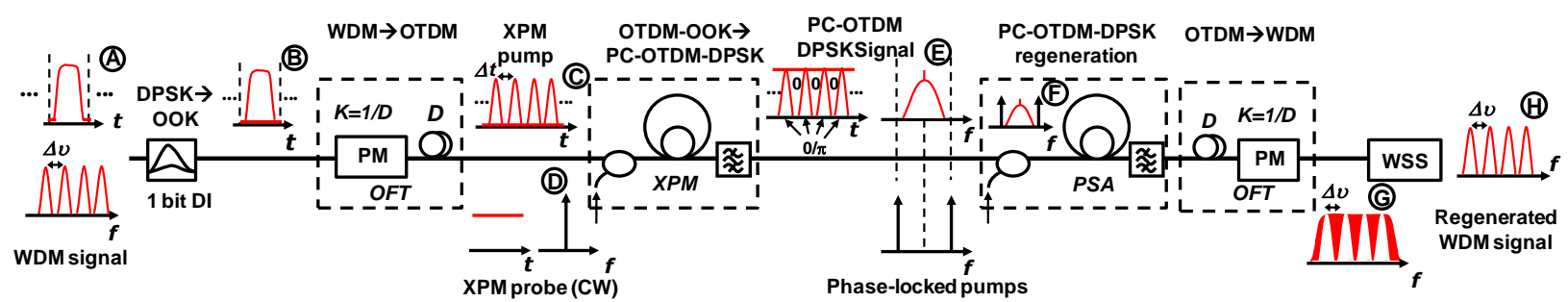

Fig. 1: Principle of all-optical WDM regeneration of DPSK signals 


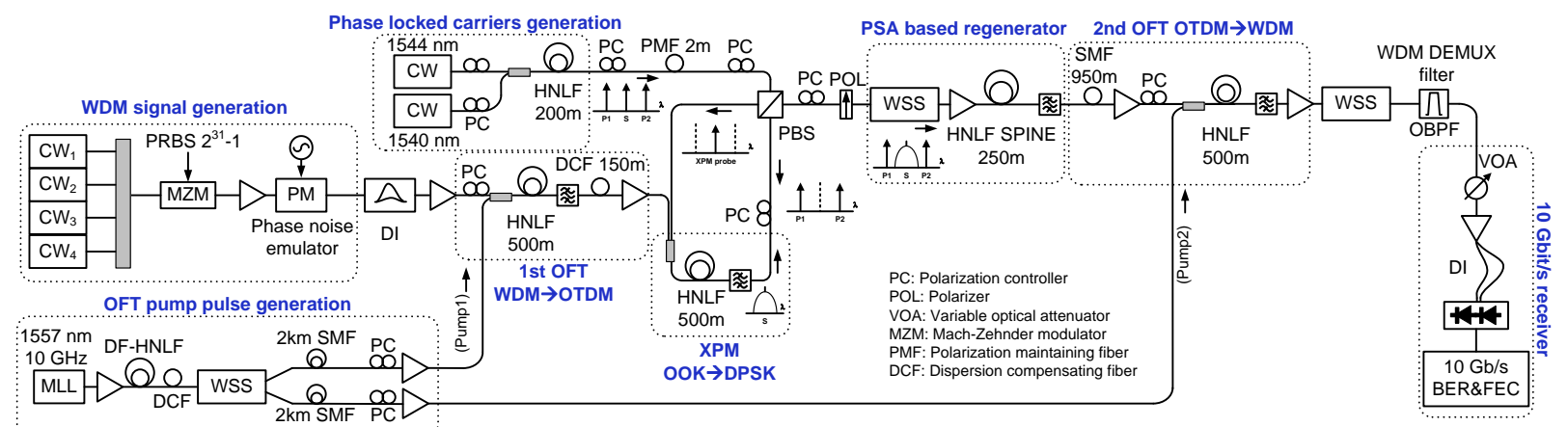

Fig. 2: Experimental setup of $4 \times 10 \mathrm{Gbit} / \mathrm{s}$ DPSK WDM regeneration

phase-locked pumps, the obtained PC-OTDM DPSK signal $(\mathbf{F})$ is sent to a HNLF to achieve phase sensitive amplification for phase regeneration. The second OFT is then used to convert the regenerated PC-OTDM channel back to WDM channels $(\mathbf{G})$. Note that after the OFT, the unmodulated $\mathrm{CW}$ parts are finally removed by a wavelength selective switch (WSS) (H). This scheme allows numerous WDM channels to be regenerated in a single PSA without detrimental interchannel cross-talk, since there are no channels overlapping in time.

\section{Experimental setup and results}

The experimental setup is shown in Fig. 2. The WDM signal source consists of 4 distributed feedback laser diodes (DFB-LDs), centered from 1548.8 to $1551.2 \mathrm{~nm}$ with a $100 \mathrm{GHz}(0.8 \mathrm{~nm})$ spacing. The outputs of the $\mathrm{CW}$ lasers are then DPSK modulated with a $10 \mathrm{Gbit} / \mathrm{s} 2^{31}-1$ PRBS in a Mach-Zehnder modulator. Phase noise is emulated on all DPSK WDM channels using a phase modulator driven by a $4.7 \mathrm{GHz}$ unsynchronized sinusoidal RF signal. The modulation index is 0.59 . A 1 bit (100 ps) DI is used to simultaneously convert all DPSK WDM channels to OOK channels. The obtained OOK WDM signal is sent to the first OFT for WDM to OTDM conversion. The quadratic phase modulation is implemented based on a fourwave mixing (FWM) process in a HNLF using linearly chirped rectangular pump pulses. As a source for the OFT pump pulses, we use a mode-locked laser at $1557 \mathrm{~nm}$ followed by
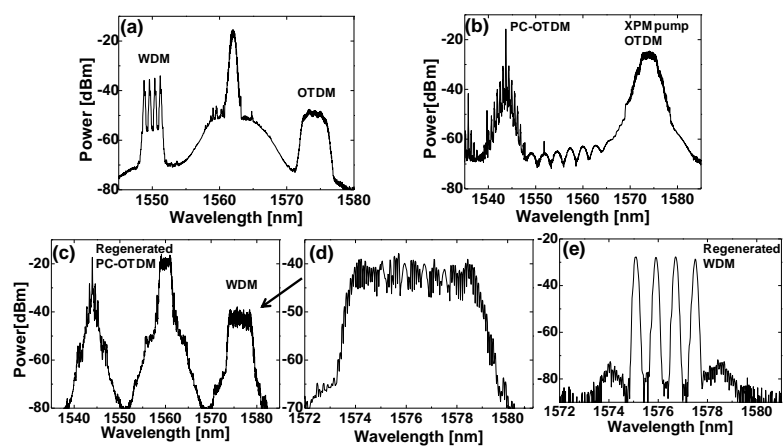

Fig. 3: Optical spectra of first OFT output(a), XPM output(b), second OFT output(c), a zoom-in on the idler(d) and the final regenerated WDM signal after WSS (e). spectral broadening in DF-HNLF. Both pumps (1st and 2nd OFT) are obtained through filtering in a WSS followed by $2 \mathrm{~km}$ SSMF propagation, yielding a chirp rate $K=0.048 \mathrm{ps}^{-2}$ suitable for conversion between a 13 ps temporal spacing and a $100 \mathrm{GHz}$ frequency grid. The output of the first OFT is shown in Fig. 3a. The generated idler is the obtained OTDM-OOK signal.

To achieve a suitable set of phase-locked pumps with the signal carrier for PSA, we use a phase-locking-free scheme based on an XPM loop ${ }^{5}$. Three phase-locked carriers P1 (1540 $\mathrm{nm})$, S (1544 nm) and P2 (1548 nm) are generated based on FWM in a $200 \mathrm{~m}$ HNLF. The polarization of carrier $S$ is rotated $90^{\circ}$ by aligning the input polarization at $45^{\circ}$ with respect to the primary polarization axis of a $2 \mathrm{~m}$ polarization maintaining fiber (PMF), where the PMF birefringence was matched to the spacing of the three carriers. After a polarizing beam splitter (PBS), the carrier S and P1, P2 are sent in opposite directions inside an optical loop containing $500 \mathrm{~m}$ of HNLF. The obtained OTDM-OOK signal is coupled into the loop as XPM pump in the same direction with $S$, and by carefully adjusting the pump power, the carrier $S$ is DPSK modulated optically (Fig. 3b). The counter propagating pumps experience only a constant phase offset. After XPM, the two pumps $\mathrm{P} 1, \mathrm{P} 2$ and the DPSK signal $\mathrm{S}$ are aligned to exit the loop using a polarization controller (PC). Here, $S$ is still orthogonally polarized to $\mathrm{P} 1$ and $\mathrm{P} 2$, and a polarizer is used to project them onto the same plane of polarization. A WSS is used to equalize the pumps, remove out of band noise and adjust the power and phase of $S$ relative to the pumps $P 1$ and P2, in order to optimize the PSA performance. The signal $S$ and pumps $\mathrm{P} 1, \mathrm{P} 2$ are amplified to $23.5 \mathrm{dBm}$, and launched into a $250 \mathrm{~m} \mathrm{HNLF}$ to achieve phase sensitive amplification with stable phase-matching for improved nonlinear efficiency (HNLF-SPINE) $)^{5}$.

After the PSA, the regenerated OTDM signal is sent to the second OFT for OTDM to WDM conversion. The output of the second OFT is 


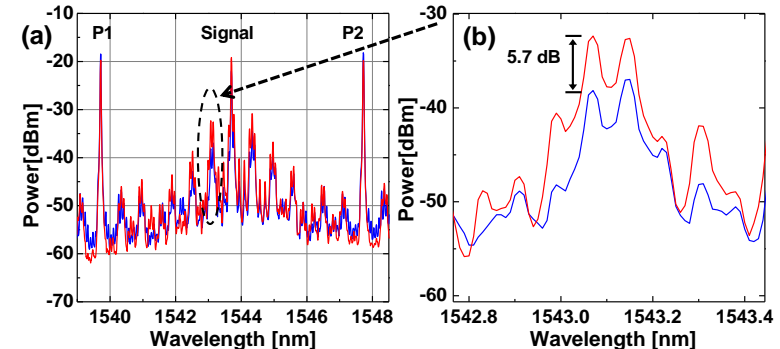

Fig. 4: (a) spectra at the PSA output for maximum (red) and minimum (blue) output signal power, (b) a zoom-in on the signal.

shown in Fig. 3c. A zoom-in on the idler is also shown in Fig.3d, where 4 obtained WDM channels are observed with some unwanted frequency components converted from the unmodulated CW carrier. The unwanted frequency components are removed by a WSS, resulting in 4 regenerated WDM channels (Fig. 3e). Finally, after WDM demultiplexing, the BER of each channel is measured in a 10-Gbit/s preamplified DPSK receiver including a DI and balanced photo-detection.

The PSA output spectrum for maximum and minimum output signal power is shown in Fig. $4 \mathrm{a}$, by sweeping the relative phase between the pumps and signal. To avoid the influence from the unmodulated $\mathrm{CW}$ component, the phase sensitive extinction ratio $5.7 \mathrm{~dB}$ is measured at the side lobe as shown in Fig. 4(b). As all WDM channels are modulated by the same PRBS pattern, strong side lobes can be observed on the signal spectrum. Fig. 5a shows the BER performance of 4 regenerated phase noise degraded DPSK WDM channels. For reference, the back to back BER curves of the WDM
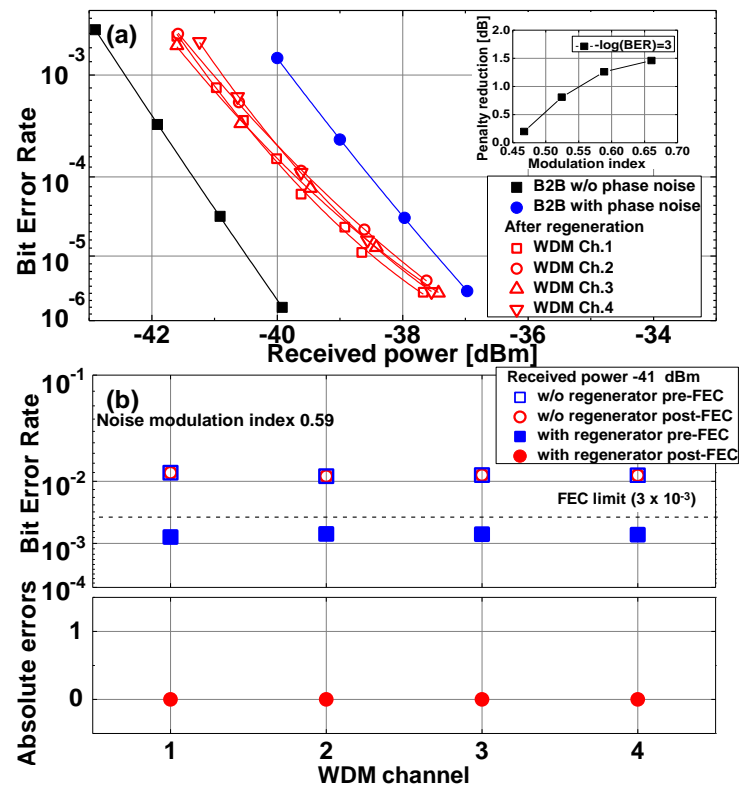

Fig. 5: Experimental results, (a) BER performance of the regenerated WDM channels, inset: penalty reduction VS. different modulation index of the phase noise. (b) pre-FEC and post-FEC BER performance for all 4 regenerated WDM channels at a received power of $-41 \mathrm{dBm}$. channels with and without phase noise are also plotted in Fig. 5a (only a single channel is plotted for both cases since all WDM channels have nearly identical $B E R$ performance). The power penalty is $2.6 \mathrm{~dB}$ at $\mathrm{BER}=10^{-3}$, when phase noise is added. After WDM regeneration, the power penalty is reduced by $1.3 \mathrm{~dB}$ for all WDM channels. The inset of Fig. $5 a$ shows the power penalty reduction versus different modulation index of the phase noise at $\mathrm{BER}=10^{-3}$, which indicates a better regeneration performance for higher phase noise. We also measured the absolute pre-FEC and post-FEC errors for all WDM channels using a FEC module at a low received power level $-41 \mathrm{dBm}$ (Fig. 5b). Without the regenerator, the BERs of all 4 WDM channels were around $10^{-2}$ due to the added phase noise, making it impossible to recover the data by FEC. With the regenerator, the BER was improved to below the FEC limit $3 \times 10^{-3}$ for all regenerated channels. The FEC module then properly recovers the data making us unable to measure any errors after FEC for all 4 regenerated WDM channels.

\section{Conclusion}

We have proposed a novel all-optical WDM regeneration scheme for DPSK signals based on optical Fourier transformation and phase sensitive amplification. Four 10-Gbit/s phase noise degraded DPSK WDM channels were regenerated simultaneously for the first time, achieving $1.3 \mathrm{~dB}$ power penalty improvement and error free performance with FEC for all channels. In principle, the system complexity of the proposed scheme does not scale with the number of WDM channels, making the scheme attractive for scaling to large number of WDM channels.

\section{Acknowledgment}

OFS Denmark Aps, Danish Research Council FTP project TOR (ref. no. 12-127224)

\section{References}

[1] L. Provost et al., "Optical WDM regeneration: Status and future prospects," Proc. OFC, OWD7, San Diego (2009).

[2] R. Slavík et al., "Practical issues and some lessons learned from realization of phase sensitive parametric regenerators," Proc. OFC, OW3C4, Los Angeles (2012).

[3] P. Guan et al., "All-Optical Ultra-High-Speed OFDM to Nyquist-WDM Conversion," Proc. OFC, W3C.6, Los Angeles (2015).

[4] H. Hu et al., "640 GBd phase-correlated OTDM NRZOOK generation and field trial transmission," JLT., Vol. 31, no. 4, p. 696 (2013)

[5] N. K. Kjøller et al., "A Novel Phase-Locking-Free Phase Sensitive Amplifier based Regenerator," Proc. OFC, W4C.2, Los Angeles (2014). 